\title{
Sampling techniques for adult Afrotropical malaria vectors and their reliability in the estimation of entomological inoculation rate
}

\author{
L.E.G. MBOERA \\ National Institute for Medical Research, P.O. Box 9653, Dar es Salaam, Tanzania; \\ E-mail:lmboera@nimr.or.tz
}

\begin{abstract}
Various entomological indicators and sampling techniques are used to monitor and evaluate the impact of many vector control interventions. A number of methods have been used in sampling mosquitoes for the purpose of estimating the entomological inoculation rate (EIR) and each is subject to some bias or shortcomings. It was the aim of this paper to critically evaluate the most common mosquito sampling techniques in relation to their reliability in the estimation of EIR. The techniques include man-landing, light trap, light trap/bednet combination and odour-baited traps. Although man-landing technique is the most reliable, it however, expose the catcher to mosquito-borne infections. On the other hand, light traps have been found to capture mosquitoes with higher sporozoite rates as compared to those from human bait catch thus leading to an overestimation of EIR. From an epidemiological point of view, the use of light-trapbed net combination is an approach that is more meaningful than using light trap alone because, a light trap functions more efficiently when placed near the normal flight paths of mosquitoes such as inside huts or under the eaves. Unfortunately, it has been shown that estimates of EIR are influenced by trap position, hence affecting the number caught and the sporozoite rates. A variety of bednets have been used to sample mosquitoes attracted to man. Studies have shown that bednet traps normally catch fewer mosquitoes than do human baits outside them. Although the collections by indoor resting technique give a good estimate of the mean house density in a given area, they may not necessarily give a good estimate of EIR. Thus the development of improved sampling systems based on an improved understanding of hostoriented behaviour is needed. Moreover, there is need to standardise all the sampling techniques in use to enable us make valid comparisons between various studies done by different people and in different areas. In this article, the inherent limitations of conventional mosquito sampling techniques when used in estimating the EIR are discussed.
\end{abstract}

Key words: malaria vector, sampling, traps, entomological inoculation rate, Africa

\section{Introduction}

Vector control is an essential component of malaria control programmes. In attempting to control malaria targeting the vector through insecticidetreated mosquito nets, biological control of larvae and adults, environmental management and indoor spraying with residual insecticides, it is important to evaluate the impact of intervention measures on the immediate or direct target as well as their relative contribution to the overall effect on both the vector population and the disease (WHO, 1995). To obtain such information, reliable indicators and sampling techniques have to be developed.

Various entomological indicators and sampling techniques are used to monitor and evaluate the impact of any vector control intervention (WHO, 1975a,b). The choice of method is of paramount importance in sampling mosquito populations (Service, 1977). The entomological parameters being studied and the behaviour of the mosquito species being sampled determine the choice of a method. In any particular investigation, there should be a careful selection of the most appropriate methods so that the most meaningful and pertinent information is collected.

In entomological terms, success of any vector control method can be defined as a reduction in the frequency at which people are bitten by infectious mosquitoes- the entomological inoculation rate (EIR) (Lines et al., 1991). The EIR, i.e. the product of the sporozoite rate and the man-biting rate, is the most important epidemiologically meaningful estimate of human-vector contact. Thus, assessments of man-biting rate and the proportion of malaria-infected mosquitoes are important components of malaria field studies (Davis et al., 1995). The estimation of actual man-biting rates, however, remains difficult (Service, 1993). Many techniques have been used in sampling mosquitoes for the purpose of estimating the EIR and each is subject to some bias or shortcomings. In this paper, the most common mosquito sampling techniques are discussed in relation to their reliability in the estimation of EIR. 


\section{Man-landing}

Man-landing catches are currently the most reliable measure of human-vector contact for calculating the EIR. In a typical man-landing catch, 1-3 people acting as both baits and catchers sit down at a selected site and aspirate mosquitoes landing on their exposed limbs. The advantage of this method is that it directly measures the biting rate of anthropophagic mosquitoes considered to be representative of the vector population responsible for malaria transmission (Davis et al., 1995). This is because the mosquitoes are caught in precisely the act in which we are interested- the act of biting a person (Lines et al., 1991). Unfortunately, this method has some major disadvantages and is facing opposition. First, an all-night man-landing catch requires well-motivated staff and close supervision if the results are to be reliable (WHO, 1995). Second, it raises some ethical issues because the occupational exposure to host-seeking anophelines may place the collectors at an increased risk of being bitten by infected mosquitoes (Service, 1977). The increasing numbers of drug resistant parasite strains further compounds this (Rubio-Palis, 1996). Third, and perhaps most important, there is the element of collector subjectivity as a result of variability in human attractiveness and skill in catching mosquitoes and thus it is difficult to standardize the estimates based on biting catches. In addition, this method is cumbersome and labour intensive. There is therefore, a need to search for a satisfactory method of sampling Afrotropical malaria vectors that would reduce the need to use man-landing catches. The possibility of making valid biting estimates from artificial traps (or any other sampling trapping device) is highly desirable. The important requirement is that the relationship between trap and man-landing catches be calibrated to suit local conditions (Lines et al., 1991).

\section{Light trap}

In search for a substitute to the human landing catch, the reliability of light traps in obtaining EIR has been evaluated under different settings and has shown varying degrees of success (Service, 1970; Chandler et al., 1975; Joshi et al., 1995). Light traps were introduced into the USA for mechanical sampling of mosquitoes following realisation of the variation between the attractiveness of different men to mosquitoes (Headlee, 1928). Since then, various designs and applications of light traps have been used in sampling anthropophagic mosquito populations in order to indirectly estimate human biting rates and hence EIR (Service, 1993).

Light traps have proved exceptionally useful for catching Aedes taeniorhynchus, Ae. nigromaculis, Culex tarsalis and Cx. t. surmmorus (Service, 1977). Of the light traps, the standard Centers for Disease Control (CDC) miniature light trap (Sudia \& Chamberlain, 1962) has been found to be fairly good for sampling endophagic malaria and bancroftian filariasis vectors in Africa (Odetoyinbo, 1969). For the general purpose of sampling the mosquito population in an area, light traps are useful as they catch more mosquitoes than man-landing catch (Davis et al., 1995; Shiff et al., 1995) and provide more information on the specific composition of the Anopheles fauna during a period of high mosquito density (Joshi et al., 1975). However, for malaria transmission monitoring purposes, there is need to trap as many mosquitoes as possible. On the contrary, it is only necessary to trap the equivalent number of mosquitoes that would normally be attracted to an average human host in a night, i.e. mosquitoes of the same species and same age range, and a similar proportion that are infected with malaria parasites.

In most studies, light traps have been found to capture mosquitoes with higher sporozoite rates as compared to those from human bait catch (Faye et al., 1992; Mbogo et al., 1993) thus leading to an overestimation of EIR. This can be accounted for by the tendency of light traps to attract and capture resting mosquitoes. Resting mosquitoes have been shown to have a higher sporozoite rate than hostseeking ones (Petrarca et al., 1991). Moreover, some studies have shown lower parity rates in mosquitoes sampled by light traps in comparison to those from man-landing catch (Lines et al., 1991).

Several authors have regressed light trap catches on standard man-landing catch in an attempt to find a functional relationship that may be used to infer biting rates from the number of mosquitoes 
Table 1: Comparison of mosquito sampling by light trap and man-landing catches in Africa

\begin{tabular}{llll}
\hline Species & LT:MLC* & Location & Reference \\
\hline An. gambiae s.l. & $1.5: 1$ & Muheza, Tanzania & Lines et al. (1991) \\
An. gambiae s.l. & $1.18 ; 1$ & Bagamoyo, Tanzania & Davis et al. (1995) \\
An. gambiae s.l. & $1.08: 1$ & Noungou, Burkina Faso & Costantini et al. (1998) \\
An. funestus & $1.5: 1$ & Muheza, Tanzania & Lines et al. (1991) \\
An. funestus & $1.32: 1$ & Bagamoyo, Tanzania & Davis et al. (1995)
\end{tabular}

*LT: MLC $=$ light trap: man landing catch ratio

caught in the traps (Rubio-Palis \& Curtis, 1992; Githeko et al., 1994). However, there are suggestions on theoretical grounds, that inferences based on regression analysis may be misleading (Lines et al., 1991). Following this suggestion, some studies have shown variations in the relationship of human biting catches with light trap catches (Table 1). In common with most trapping techniques, the quantity and quality of the catch depends on the trap design, weather conditions and other variables, such as trap location (Service, 1993; Mboera et al., 1998). This is a clear indication that, light traps while useful in some ways, do not represent the true manbiting mosquito population.

Although light traps have been employed in the study of the various aspects of mosquito biology and ecology, their use in obtaining meaningful estimates of the EIR is limited, as it is difficult to interpret the numbers caught in terms of mosquito-man contact. This is because the mechanism of attraction of mosquitoes to light trap is not well understood and may only be loosely related to the act of feeding on a human being.

\section{Light trap/bednet combination}

Light trap catches improve when traps are located close to bednets occupied by humans (Garrett-Jones \& Magayuka, 1975; Lines et al., 1991; Shiff et al., 1995; Mboera et al., 1998). Clearly, the attraction of host seeking mosquitoes to the person inside the bednet and the prevention of feeding by the net increase their chance of capture by the light traps. Although the exact trapping mechanism of this light trap/bednet combination remains unknown, it is possible that mosquitoes which persistently attempt to penetrate the bednet and exploring all their way around it would eventually come close to the trap and be caught. From an epidemiological point of view, this approach might be more meaningful than using light trap alone, because without bednets, it is difficult to interpret the number caught in terms of mosquito-man contact. In addition, light trap may function more efficiently when placed near the normal flight paths of mosquitoes such as inside huts or under the eaves (Odetoyinbo, 1969).

The unique advantage this method may have over using light traps on their own is that occupied bednets and man-landing catch attract similar cohorts from the population. However, closer observations of this light trap-bednet system revealed that the catch size and parity rates of hostseeking mosquitoes differ according to the trap position in relation to the host occupying the bednet (Mboera et al., 1998). This could be due to the fact that mosquitoes show a preference for biting certain body parts (Knols, 1996). It follows that estimates of EIR will probably be influenced by trap position as this affects the number caught and the sporozoite rates (which will increase with higher parity rates). The estimation of the EIR will be affected as follows:

$$
\begin{gathered}
\mathrm{EIR}=m \times a \times p ; \text { where: } \\
m=\text { relative density } \\
a=\text { human biting rate } \\
p=\text { sporozoite rate }
\end{gathered}
$$

Following the results by Mboera et al. (1998): (i) $m$ will be higher when the light trap is positioned high above the foot-end of the bed and will raise the EIR; (ii) $p$ will also be higher at the same position due to a higher parity rate (older mosquitoes) and will thus 
raise the EIR. From this example it is clear that significant variations in the EIR will be obtained if CDC light traps are not used in a standardised manner.

Although various workers place the light trap as close to the host as possible, no attempts has been made to standardise the trap position, the trap distance from the floor or the trap distance from the bednet. Another problem is that in most of these studies, traps were switched on by sleepers (Lines et al., 1991; Costantini et al., 1998), and hence, the timing off trap operation cannot be ascertained. For instance, in the study by Costantini et al. (1998), traps were switched on at $21.00 \mathrm{~h}$ and sleepers were instructed to switch off at $05.00 \mathrm{~h}$. A standardised light trap operation time is important as people retire to bed and get up at different time of the night and morning, respectively. This may affect the number of mosquito attracted and consequently caught in the trap. Moreover, switching off traps as early as $05.00 \mathrm{~h}$ may result in missing a good sample of mosquitoes that bite late in the early morning hours. Standardised use of this light trap/bednet sampling method is needed if meaningful estimates of EIR are to be obtained. One important observation to make is that whole human odour attracts a similar number of mosquitoes as occupied bednet (Mboera et al., 1997). This can therefore permit the standardisation of the light trap/bednet system.

\section{Bednet trap}

A variety of bednets have been used to sample mosquitoes attracted to man or other hosts. Usually, a man sleeps under a bednet that is either raised a few centimetres from the ground or has one or two panels rolled back to horizontal slits or tears to provide ingress of hungry mosquitoes (Service, 1977). The person acting as bait can be enclosed within an inner net to protect him from mosquito bites. The reliability of a bednet trap catch in the estimation of EIR as compared to that obtained from the human-bait catch is subject to debate.

A number of studies have shown that bednet traps normally catch fewer mosquitoes than do human baits outside them (Service, 1993; Le Goff et al., 1997). However, mosquitoes sampled by bednet traps have parity rates and sporozoite indices that compare favourably with those obtained from the human-bait catches (Le Goff et al., 1997). Perhaps, this observation is due to the fact that the hostseeking mosquitoes are attracted to the odours emanating from the host in the bednet trap and therefore the catch from such a method may represent a reliable cross section of the host-seeking population.

As mentioned earlier, what is needed is the equivalent numbers of mosquitoes that would normally bite an average person in a night. In addition this method also exposes the baits to mosquito bites and attempts to use a double bednet have not yielded satisfactory results (Service, 1993; Le Goff et al., 1997). This calls for modification of such bednet traps. Such a modification can be accomplished only if the behaviour of the mosquitoes around occupied bednets is well understood. Equipped with a detailed knowledge of the behaviour of mosquitoes around bednets, it is possible to modify the nets so that they can give maximum protection to the users. In addition, a modification can be made so that the nets may also serve as sampling devices for the actual hostseeking mosquitoes. The main idea is to utilise host attraction to develop a bednet trap for sampling Afrotropical malaria vectors while at the same time safeguarding against the risk of exposure to infective bites. In addition to using a light trap next to a bednet, the modification of bednets for sampling host-seeking mosquitoes has been investigated using the so-called Mbita trap (Mathenge et al., 2002).

\section{Mbita trap}

The Mbita trap, described by Mathenge et al. (2002), consists of an entry-no return device whereby human are used as bait but cannot be bitten. The trap consists of a modified conical bednet made of white cotton cloth that concentrates in its upper part the convection current and various odours producted by the human bait; the apex is made of netting and forms a funnel with a small round hole $(50 \mathrm{~mm}$ in diameter) at its base that permits the entrance of mosquitoes but impedes their escape; a netting panel is fixed halfway up the net to separate the upper mosquito chamber from the lower human chamber (Mathenge et al., 2002). The authors claimed the trap to be relatively inexpensive 
to produce, requires minimum maintenance and is simple to use.

Mathenge et al. (2002) provide evidence of the efficiency of Mbita trap in sampling laboratory reared An. gambiae, released in a screen-walled greenhouse in Western Kenya. When compared side-by-side with similar samples of mosquitoes, the Mbita trap caught $43 \pm 10 \%$ of the number caught by human landing catches. However, in a recent study in Madagascar, Laganier et al. (2003) found that the number of mosquitoes collected was 15.4 per human-night and 1.0 per trap-night with an efficiency of $0.06 \%$ for the Mbita trap versus human landing. The number of anophelines was 10.30 per human-night and 0.55 per trap-night, i.e. an efficiency of $5.3 \%$. This efficiency was $10 \%$ for indoor An. funestus, 24\% for outdoor An. funestus, and 3\% for An. arabiensis. Laganier et al. (2003) concluded that the efficiency of Mbita trap compared to human landing collection is very poor and unreliable as compared to classic human landing catches. The findings in Madagascar were in complete contradiction with previous studies in Kenya (Mathenge et al., 2002).

In a later study in rice irrigation in western Kenya, Mbita trap was found to catch $17 \%$ and $60 \%$ of the number of An. arabiensis and An. funestus species caught in the human landing collections, respectively (Mathenge et al., 2004). A consistency in sampling proportionality between Mbita trap and human landing catch was observed for $A n$. arabiensis. Interestingly, in this study, CDC light caught about $60 \%$ and $120 \%$ of the number of $A n$. arabiensis and An. funestus caught in the human landing collections, respectively. The authors admitted that the Mbita trap is less sensitive than either the human landing catch or the CDC light trap.

\section{Indoor resting}

Several malaria vectors are known to rest in houses before and/or after feeding on a man. Collection of indoor resting mosquitoes is usually done using aspirators or by knockdown pyrethrum spray catches (Service, 1993). Although collections by these methods give a good estimate of the mean house density in a given area, they may not necessarily give a good estimate of EIR. This is due to the fact that sampling indoor-resting mosquitoes tends to miss the mosquitoes that leave the house immediately after feeding, and may include those that enter after feeding outdoors on other hosts. With species that normally feed and rest indoors, the presence of an irritant insecticide such as DDT may drive the mosquitoes to rest outside, without preventing them from first feeding indoors. Thus indoor resting-catches can give rise to a false impression of effectiveness of a control measure (Lines et al., 1991). Moreover, the procedure is cumbersome to house occupant when collection is taking place.

\section{Future prospects of sampling mosquitoes in Africa}

Tent traps: During the past decade, polyvinylchloride (PVC) tents have recently been employed in studying host-seeking An. gambiae, An. funestus and Cx. quinquefasciatus (Mboera et al., 1997; Knols et al., 1995; Mboera \& Takken, 1999). Mosquitoes could enter the tents through slits made just beneath the roof on both sides of the tents. Two exit traps (Muirhead-Thomson, 1948) are fitted to the walls of the tents. Inside, an unimpregnated rectangular bednet is hung, next to which a standard miniature CDC light trap (Sudia \& Chamberlain, 1962) is operated.

This type of sampling tool provide an indoor and outdoor environment and thus useful in sampling both the endophagic-endophilic and endophagic-exophilic mosquitoes. In addition, tenttraps can be moved from one side of the village to another thus providing a standardised type of indoor setting, normally difficult to obtain using houses. Many houses in Africa differ in construction, architectural design, size, wall painting, and size of eaves or ventilators. The effects of these house variations on mosquito catches have not been studied in detail. Nonetheless, some studies have shown that vector abundance and biting densities are influenced by factors such as local variations in climate, topology, house design, the proximity of houses to mosquito breeding sites and colour of the landing sites (Lindsay \& Snow, 1988; Lindsay et al., 1995; Mutinga et al., 1995). Therefore, employment of sampling tools such as tent-traps would facilitate critical work on many aspects of mosquito 
behaviour and improve our understanding of mosquito-host interaction. Despite the prospective merits and usefulness of tent-traps, they have never been employed in routine studies of malaria transmission in Africa.

Odour-baited traps: Odour-mediated resourcelocating behaviours of the anthropophilic mosquitoes have been studied in detail during the past decade (Knols, 1996; Costantini, 1996; Mboera, 1999). Although many individual aspects of these behaviours are little understood, there are reasons to believe that studies towards some of these processes will open the way for manipulation of these behaviours towards mosquito monitoring and control. For example, despite the minor role of carbon dioxide in attracting anthropophilic mosquitoes to their hosts, carbon dioxide-baited traps may collect larger numbers of An. gambiae, An. funestus and Cx. quinquefasciatus (Knols et al., 1995; Mboera, 1999) than most of the already identified mosquito kairomones such as 1-octenol3-ol and acetone.

Efforts to sample anthropophilic mosquitoes in outdoor situation have been found to be difficult when using odour-baited CDC light traps. Nonetheless, Costantini et al. (1993) have shown that it is possible to attract mosquitoes into a device baited with cues associated with host location using odour-baited entry traps (OBET). The OBET successfully caught similar numbers of $A n$. gambiae, s.l. as a CDC light trap next to a human baited bednet, and about $33 \%$ of an all-night human biting catch nearby. To date, many studies have shown that baiting traps with whole human odours increases the catch of mosquitoes (Knols et al., 1995; Costantini, 1996; Costantini et al., 1993; Knols et al., 1998). Thus the development of improved sampling systems based on an improved understanding of host-oriented behaviour is needed.

Mbita trap: Studies carried out in Kenya and Madagascar have shown that Mbita trap is less sensitive than either human landing catch or the CDC light trap (Mathenge et al., 2002; 2004; Laganier et al., 2003). However, the trap has been found to catch more mosquitoes over a longer period, larger number of sampling sites and may be useful for enabling community members in collecting samples that are representative of the overall vector population at a less cost (Mathenge et al., 2002). Nonetheless, the authors caution that Mbita is unlikely to work in all epidemiological settings and therefore more mosquito behavioural studies need to be carried out in order to gain more insight to guide further development of mosquito sampling and control tools.

\section{Conclusion}

There are many limitations associated with the traditional methods of sampling malaria vectors. Such limitations can only be overcome if the behaviours of the vectors, particularly the interaction with the host, are well understood. Mosquito traps that incorporate human body odour would be environmentally friendly. Moreover, they may provide an alternative approach to mosquito sampling and control. It is therefore necessary that future studies focus on mosquito olfaction as this may result in the development of a new low-tech trap for community use. The use of tent traps and the possibility of modifying bednets in order to sample host-seeking mosquitoes need serious consideration. Lastly, and perhaps most important, there is need to standardise all the sampling techniques in use to enable us make valid comparisons between various studies done by different people and in different areas. Despite the risk of acquiring mosquito-borne infections, human landing catch remains the golden standard in estimating mosquito density and entomological inoculation rate. The human landing catch should therefore, be maintained as the standard reference technique for use in calibrating new methods for sampling the human biting population of mosquitoes.

\section{Acknowledgements}

Drs Helen Pates-Jamet and Stephen Magesa are thanked for reviewing the earlier version of the manuscript.

\section{References}

Chandler, J.A., Highton, R.B. \& Hill, N.M. (1975) Mosquito of the Kano Plain, Kenya I. Results of indoor collections in irrigated and nonirrigated areas using human bait and light traps. Journal of Medical Entomology 12, 504-510. 
Costantini, C. (1996) Behavioural Studies on West African Malaria Vectors in the Field. Ph.D Thesis, 228pp. Imperial College of Science, Technology and Medicine, University of London, UK.

Costantini, C., Sagnon, N.F., Sanogo, E., Merzagora, L. \& Coluzzi, M. (1998) Relationship to human biting collections and influence of light trap and bednet in CDC lighttrap catches of West African malaria vectors. Bulletin of Entomological Research 88, 503511.

Costantini, C., Gibson, G., Brady, J., Merzagora, L. \& Coluzzi, M. (1993) A new odour-baited trap to collect host-seeking mosquitoes. Parassitologia 35, 5-9.

Davi s, J.R., Hall, T., Chee, E.M., Majala, A., Minjas, J. \& Shiff, C.J. (1995) Comparison of sampling of anophelines mosquitoes by light trap and human-bait collections indoor in Bagamoyo, Tanzania. Medical and Veterinary Entomology 9, 249-255.

Faye, O., Diallo, S., Gaye, O., Ndir, O \& Faye, O. (1992) Comparative efficacy of the use of CDC light traps and humans to sampling Anopheles populations. Results obtained in the area of Bigona. Bulletin of the Society of Pathology Exotica 85, 185-189.

Garrett-Jones, C. \& Magayuka, S.A. (1975) Studies on the natural incidence of Plasmodium falciparum and Wuchereria infections in Anopheles in rural East Africa: I. Assessment of densities by trapping hungry female Anopheles gambiae Giles species A. WHO/MAL/75.851, WHO/VBC/75.541.

Githeko, A.K., Service, M.W., Mbogo, C.N., Atieli, F.A. \& Juma, F.O. (1994) Sampling Anopheles arabiensis, An. gambiae, sensu lato and An. funestus (Diptera: Culicidae) with CDC light trap near a rice irrigation area and a sugarcane belt in western Kenya. Bulletin of Entomological Research 84, 319-324.

Headlee, T.J. (1928) The development of mechanical apparatus for detecting the presence of mosquitoes in various localities. Proceedings of the New Jersey Mosquito Extermination Association 15, 160-168.

Joshi, G.P., Service, M.W. \& Pradhan, G.D. (1975) A survey of species A and B of the Anopheles gambiae Giles complex in the Kisumu area of Kenya prior to insecticidal spraying with OMS43 (fenitrothion). Annals of Tropical Medicine \& Parasitology 69, 91-104.

Knols, B.G.J. (1996) Odour Mediated Host-seeking Behaviour of the Afro-tropical Malaria Vector Anopheles gambiae Giles. PhD Thesis, 213pp. Wageningen Agricultural University, The Netherlands.

Knols, B.G.J., De Jong, R. \& Takken, W. (1995) Differential attractiveness of isolated humans to mosquitoes in Tanzania. Transactions of the Royal Society of Tropical Medicine and Hygiene 89, 604-606.

Knols, B.G.J., Mboera, L.E.G. \& Takken, W. (1998) Electric nets for studying odour-mediated hostseeking mosquitoes. Medical and Veterinary Entomology 12, 116-120.

Le Goff, G. Carnevale, P., Fondjo, E. \& Robert, V. (1997) Comparison of three sampling methods of man-biting anophelines in order to estimate the malaria transmission in a village in south Cameroon. Parasite 4, 75-80.

Lindsay, S.W. \& Snow, R.W. (1988) The trouble with eaves: house entry by vectors of malaria. Transactions of the Royal Society of Tropical Medicine and Hygiene 82, 645-646.

Lindsay, S.W., Schellenberg, J.R., Zeiler, H.A., Daly, R.J., Salum, F.M. \& Wilkins, H.A. (1995) Exposure of Gambian children to Anopheles gambiae malaria vectors in an irrigated rice production area. Medical and Veterinary Entomology 9, 50-58.

Lines, J.D., Curtis, C.F., Wilkes, T.J. \& Njunwa, K.J. (1991) Monitoring human-biting mosquitoes (Diptera: Culicidae) in Tanzania with light-traps hung beside mosquito nets. Bulletin of Entomological Research 81, 77-84. Mathenge, E.M., Killeen, G.F., Oulo, D.O., Irungu, L.W., Ndengwa, P.N. \& Knols, B.G.J. (2002) Development of an exposure-free bednet trap for sampling of Afro-tropical mosquitoes. Medical and Veterinary Entomology 16, 6774.

Mathenge, E.M., Omweri, G.O., Irungu, L.W., Ndengwa, P.N., Walczak, E., Smith, T.A., Killeen, G.F. \& Knols, B.G.J. (2004) Comparative field evaluation of the Mbita trap, CDC light trap and the human landing catch 
for sampling of malaria vectors in western Kenya. American Journal of Tropical Medicine and Hygiene 70, 33-37.

Mbogo, C.N.M., Glass, G.E., Forster, D., Kabiru, E.W., Githure, J.I., Ouma, J.H. \& Beier, J.C. (1993) Evaluation of light traps for sampling anopheline mosquitoes in Kilifi, Kenya. Journal of American Mosquito Control Association 9, 260-263.

Mboera, L.E.G. \& Takken, W. (1999) Odour mediated host preference of Culex quinquefasciatus in Tanzania. Entomologia Experimentalis et Applicata 92, 83-88.

Mboera, L.E.G. (1999) Chemical Ecology of the Behaviour of the Filariasis Mosquito Culex quinquefasciatus Say. Ph.D Thesis, 189pp. Wageningen Agricultural University, The Netherlands.

Mboera, L.E.G., Kihonda, J., Braks, M.A. \& Knols, B.G.J. (1998) Influence of Centers for Disease Control light trap position, relative to a human-baited bednet, on catches of Anopheles gambiae and Culex quinquefasciatus in Tanzania. American Journal of Tropical Medicine and Hygiene 59, 595-596.

Mboera, L.E.G. Knols, B.G.J., Takken, W. \& della Torre, A. (1997) The response of Anopheles gambiae s.1. and A. funestus (Diptera: Culicidae) to tents baited with human odour or carbon dioxide in Tanzania. Bulletin of Entomological Research 87, 173-178.

Muirhead-Thomson, R.C. (1948) Studies on Anopheles gambiae and A. melas in and around Lagos. Bulletin of Entomological Research 38, 527-558.

Mutinga, M.J., Odhiambo, T.R., Kamau, C.C., Odulaja, A., Amimo, F.A. \& Wachira, D.W. (1995) Choice of resting sites by Anopheles gambiae (Diptera: Culicidae) in Mwea rice irrigation scheme, Kirinyaga District, Kenya. East African Medical Journal 72, 170-175.

Odetoyinbo, J.A. (1969) Preliminary investigation on the use of a light-tap for sampling malaria vectors in the Gambia. Bulletin of the World Health Organization 40, 547-560.

Petrarca, V., Beier, J.C., Onyango, F., Koros, J., Asiago, C., Koech, D.K. \& Roberts, C.R. (1991)
Species composition of the Anopheles gambiae complex (Diptera: Culicidae) at two sites in western Kenya. Journal of Medical Entomology 28, 307-313.

Rubio-Palis, Y. \& Curtis, C.F. (1992) Evaluation of different methods of catching anopheline mosquitoes in western Venezuela. Journal of American Mosquito Control Association 8, 261-267.

Rubio-Palis, Y. (1996) Evaluation of light-traps combined with carbon dioxide and 1-octen-3-ol to collect anophelines in Venezuela. Journal of American Mosquito Control Association 12, 91-96.

Service, M.W. (1970) A battery-operated light-trap for sampling mosquito populations. Bulletin of the World Health Organization 43, 635-641.

Service, M.W. (1977) A critical review o procedures for sampling populations of adult mosquitoes. Bulletin of Entomological Research 67, 343382.

Service, M.W. (1993) Mosquito Ecology. Field Sampling Methods. 2nd Ed. Elsevier Science Publishers, Essex, UK.

Shiff, C.J., Minjas, J.N., Hall, T., Hunt, R.H., Lyimo, S. \& Davis, J.R. (1995) Malaria infection potential of anophelines mosquitoes sampled by light trapping indoors in coastal Tanzanian villages. Medical and Veterinary Entomology 9, 256-262.

Sudia, W.D. \& Chamberlain, R.W. (1962) Batteryoperated light trap, an improved model. Mosquito News 22, 126-129.

WHO (1975a) Manual of Practical Entomology in Malaria. Part I. Vector Bionomic and Organization of Antimalarial Activities. World Health Organization, WHO Offset Publication, No. 13.

WHO (1975b) Manual of Practical Entomology in Malaria. Part II. Methods and Techniques. WHO Offset Publication No. 13.

WHO (1995) Vector Control for Malaria and other Mosquito-borne Diseases. Technical Report Series 857. World Health Organization, Geneva. 